\title{
Interview
}

\section{Interview with Dr.Ravindra Nanda on Current Concepts in Orthodontics}

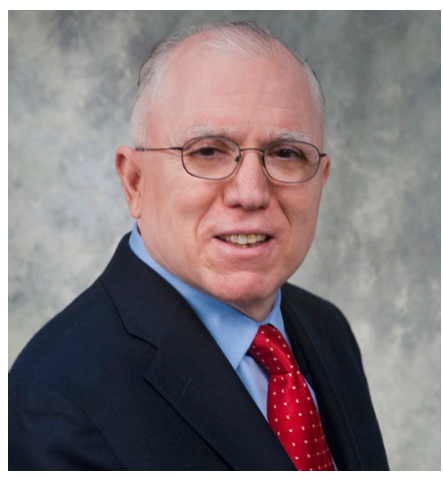

Dr.Ravindra Nanda is Professor Emeritus, Former UConn Alumni Endowed Chair and Head of the Department of Craniofacial Sciences and Division of Orthodontics, University of Connecticut, USA. Dr.Nanda, a renowned researcher and lecturer in Orthodontics, has been author of many books and scientific articles. He is on the Editorial Board of Turkish Journal of Orthodontics.

The editors of Turkish Journal of Orthodontics (TJO) did an interview with Dr.Ravindra Nanda about current concepts in orthodontics.

TJO: What has been the most important innovation of the past 20 years in orthodontics?

Dr. Nanda: I will have to say Aligners. They have changed the way we practice today.

TJO: What should be the case selection criteria in aligner treatments? And we would like to ask your opinion as an expert in biomechanics, if a paradigm shift occurred in terms of biomechanics while treating cases with clear aligners?

Dr. Nanda: My views have changed significantly over the last five years. Now I feel one can treat most of the malocclusions if done properly. I am not sold yet on aligner treatment of adolescent patients. Biomechanics principles are universal but for aligners there are quite a few differences. Major issue is deformation of aligners which makes it difficult to analyse force system the same way as we can with braces. Attachments have played a nice role in applying forces on teeth but their shape, size, site and angular positioning can affect the type of tooth movement. More studies are needed to improve our understanding of effect of non continuous forces, compliance, aligner material and attachments. Unfortunately, aligner companies are pushing treatment of even 4 and 5 years old or patients with growth discrepancies. There is no research to support these treating kids with aligners.

TJO: Do you think the evolution of the clear aligners will bring to an end to braces?

Dr. Nanda: Braces are not going anywhere. Aligners have some unresolved issues such as compliance, aligner material, and treatment of transverse and a-p discrepancies, and I can go on.

TJO: Do you use temporary anchorage devices frequently, and what should we take into consideration while using TAD's?

Dr. Nanda: I am personally retired now from active practice. I would say at our university probably $15 \%$ of patients receive TADs. In our eyes if biomechanics and differential moments are used properly, use of TADs becomes redundant. In my view TADs are ideal for en masse movements, molar intrusions, large space closures, major midline corrections and multidisciplinary patients.

TJO: Do you treat all your orthognathic surgery cases with 'the surgery first protocol'? And do you take advantage of the temporary anchorage devices in these cases?

Dr. Nanda: We feel majority of the orthognathic surgery patients can be managed by Surgery First protocol. Some patients may need minor pre surgery treatment if some teeth may prevent proper post surgical occlusion. Yes, TADs can be placed if major movement of teeth is required post surgery. 